\title{
An Adaptive Fault Locator for Transmission Lines Tapped with a Source of Generation - Using Synchronized Voltage and Current Phasors
}

\author{
Ying-Hong Lin* \\ Chih-Wen Liu* \\ Member,IEEE
}

Joe-Air Jiang**

Jun-Zhe Yang*

*: Department of Electrical Engıneering, National Taıwan Unıversity, Taipei, Taiwan

**- Private Kuang-Wu Institute of Technology and Commerce, Taipei, Taıwan

\begin{abstract}
With the advent of the high synchronism accuracy of modern Phasor Measurement Units (PMUs), an adaptive PMU-based approach using the concept of superimposed voltage and current phasors for accurately locating fault on transmission lines tapped with a source of generation is described. This paper proposes a novel faulted section discrimination index and takes the effects caused by tapped lines into account. In addition, an adaptive scheme to estimate the equivalent source impedance outside the considered transmission lines is also presented. Alternative Transients Program (ATP) simulator is used to validate the proposed fault location approach with respect to typical faults on a $100 \mathrm{~km}$. $161 \mathrm{kV}$ transmission line. The simulation results show that the accuracy of the proposed technique achieved can be up to $99.99 \%$ under different fault resistance, fault locations, pre-fault load conditions, various source impedance and various fault types.
\end{abstract}

Keywords: Phasor Measurement Units (PMUs), Fault Locator, Alternative Transients Program (ATP)

\section{INTRODUCTION}

The development of fault location techniques is very important, especially for the long lines in rough terrain, to reduce the crew repair expense and to speed up the restoration of service for power utilities. Typically, fault locators can be roughly classified into two fundamental categories: one that requires measurements from both ends of the lines and the other that only requires local data. The former is an attractive option for power utilities owing to their highly accuracy, but needs accurate synchronism on data recorders [1-3]. The later possesses the advantage in economy but suffer from certain assumptions regarding the infeed current from remote end and specified the fault types [4-5].

Recently, PMUs have been rapidly developed [6] and successfully applied to fault locators based on two-terminal data due to their high synchronism. An approach and practical implementation of PMU based fault locator has been proposed by J.-A. Jiang et al. [6-7]. Such an approach forms the basis of fault liocator using synchronized phasors.

In the past few years, owing to the privatization of electricity supply industry and dispute on the right of way in Taiwan, transmission lines tapped temporarily with a private generating plant via relatively short transmission lines were raised at Taipower system. This can drastically affect the accuracy of the fault location method described above.
In this paper, a new method to estimate the fault location under the circumstance mentioned above was proposed. The new method utilizes synchronized data from PMUs equipped at both ends of lines. Transformation of Symmetrical components is utilized to decouple effect of the inter-phase. The superimposed principle is adopted to take the effects of tapped lines into account. An adaptive scheme to estimate source impedance outside the protected lines was also proposed. The results of simulation studies to evaluate the basic performance of the proposed method are presented. The proposed method has the merit of not requiring identification of fault type in advance.

\section{BASIC PRINCIPLES}

The principles of the fault location technique are based on the assumption that the considered transmission lines are perfect transposed. Symmetrical component transformation

$$
\mathrm{T}=\frac{1}{3}\left[\begin{array}{ccc}
l & l & 1 \\
l & \mathrm{a} & \mathrm{a}^{2} \\
l & \mathrm{a}^{2} & \mathrm{a}
\end{array}\right]
$$

is adopted to resolve the coupling effect of inter-phase, where $a$ is a complex number equal to $e^{2 \pi / 3}$. Then, the three-phase transmission lines can be treated as that of single-phase ones. Since positive sequence components always appear in all types of fault events, this paper uses the positive sequence quantities to illustrate the development of the proposed algorithm.

\subsection{Review of the Two-Terminal Based Algorithm [7]}

Initially, assume that the protected transmission lines shown in Fig. 1 are without being tapped with a source of generator. Suppose that a fault occurs at point F. The two-terminal approach [7] for fault location can compute the fault location according following equation,

$$
\mathrm{D}=\ln (\mathrm{N} / \mathrm{M}) / 2 \gamma
$$

where

$$
\begin{aligned}
& N=\frac{V_{R, \text { sup }}-Z_{C} I_{R, \text { sup }}}{2}-\frac{V_{S \text {,sup }}-Z_{C} I_{S \text {,sup }}}{2} \exp (\gamma L) \\
& M=\frac{V_{S, \text { sup }}+Z_{C} I_{S \text {,sup }}}{2} \exp (-\gamma L)-\frac{V_{R, \text { sup }}+Z_{C} I_{R, \text { sup }}}{2}
\end{aligned}
$$




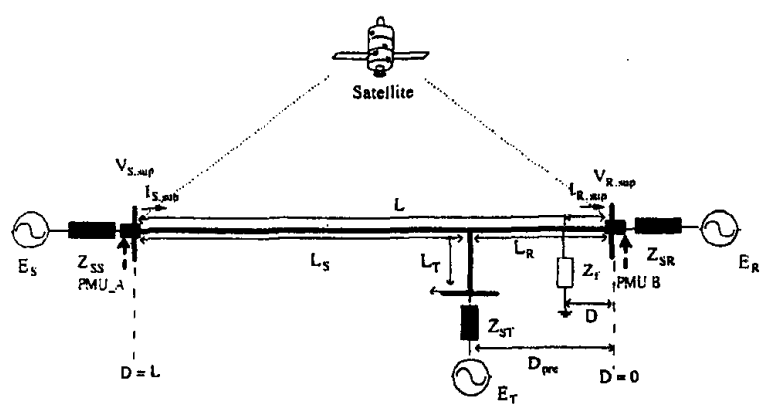

Fig. I faulted transmission lines tapped with a source of generator.

where

$V_{R \text {,sup }}$ and $V_{S \text {,sup }}$ are the superimposed synchronized voltage phasors at both ends of the lines,

$I_{R, \text { sup }}$ and $I_{S \text { sup }}$ are the superimposed synchronized current phasors at both ends of the lines,

$\gamma$ and $Z_{C} \quad$ denote the propagation constant and surge impedance of the lines, respectively.

$Z_{S S}$ and $Z_{S R}$ are the equivalent source impedance outside the protected transmission lines, respectively,

Note that the superimposed quantity can be obtained by subtracting pre-fault quantity from post-fault quantity.

\subsection{Fault Location for Transmission Lines Tapped with a} Source of Generator

The procedures for accurate fault location for transmission line tapped with a source of generator are presented in this section. The proposed fault location algorithm is based on the algorithm mentioned at section 2.1 and focus on eliminating the error caused by the tapped lines. The flowchart of the proposed method is shown in Fig.2. The phasor measurement units installed at both ends of the protected transmission line has been built with 'Global Synchronism Clock Generator (GSCG)'[6] to provide an extremely accurate and reliable external reference clock

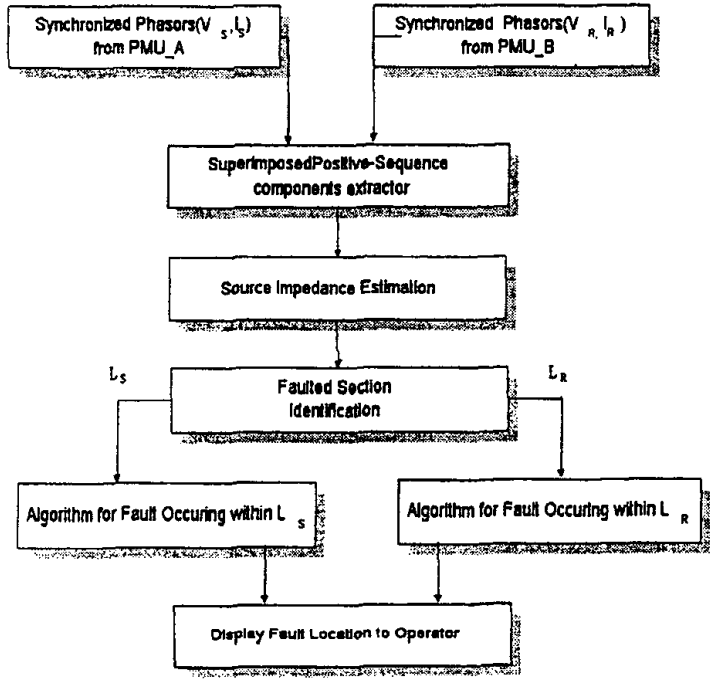

Fig.2 fault location procedures source. The performance of PMU-GSCG configuration has been verified via field-tests at Taipower $161 \mathrm{kV}$ substation that the accuracy of sampling synchronism achieved can be better than 1 microsecond [6]. This fact guarantees the measurement: synchronism of the proposed method.

The proposed method can be divided into two steps. First step is to identify the faulted section, and then an accurate fault location algorithm is applied to locate the fault.

\subsection{1 faulted section identification}

The first step of locating fault on a transmission line tapped with a source of generator is to identify the faulted section, namely, to discriminate fault occurring within either $L_{S}$ or $L_{R}$. Comparing the real part of $D_{c a l}$ with $D_{\text {pre }}$ can achieve this task. $D_{\text {pre }}$ denotes the location of tapped point and $\mathrm{D}_{\text {cal }}$ is a complex number computed from Eq.(1). The criteria of faulted section identification can be described as follows:

$\operatorname{Re}\left\{D_{\text {cal }}\right\}>D_{\text {pre }}$ : the fault section is selected as $L_{S}$

$\operatorname{Re}\left\{D_{\text {cal }}\right\}<D_{\text {pre }}$ : the fault section is selected as $L_{R}$,

$\operatorname{Re}\left\{D_{\text {cal }}\right\}=D_{\text {pre }}$ : the fault location is at tap point, where $\operatorname{Re}\{\bullet\}$ denotes real part of a complex number.

\subsection{2 fault location algorithms}

After discriminating faulted section, the fault location can be determined by utilizing the algorithm suitable to fault occurred at either $L_{S}$ or $L_{R}$. At first, considering a fault occurred within $L_{R}$, one can obtain the actual fault location $D$ away from the reference point located at receiving end $(D=0)$ by following equation:

$\mathrm{D}=\ln \left(\mathrm{N}_{\mathrm{t}} / \mathrm{M}_{\mathrm{t}}\right) / 2 \gamma$

where

$$
\begin{aligned}
N_{1} & =\frac{1}{2}\left(V_{R, \text { sup }}-Z_{C} I_{R, \text { sup }}\right)-\frac{1}{2} e^{\gamma\left(L_{R}+L_{s}\right)}\left(V_{S, \text { sup }}-Z_{C} I_{S, \text { sup }}\right) \\
& +\frac{1}{2} Z_{C} e^{\gamma I_{R}} I_{T J} \\
M_{1} & =\frac{1}{2} e^{-\gamma\left(L_{s}+L_{R}\right)}\left(V_{S, \text { sup }}+Z_{C} I_{S, \text { sup }}\right)-\frac{1}{2}\left(V_{R, \text { sup }}+Z_{C} I_{R, \text { sup }}\right) \\
& +\frac{1}{2} e^{-\gamma L_{R}} Z_{C} I_{T}
\end{aligned}
$$

and

$$
\begin{aligned}
I_{T J}= & \frac{1}{2} Z_{C}^{-1} K_{S, \text { sup }} Z_{S T}\left(e^{\gamma L_{T}}-e^{-\gamma L_{T}}\right)+ \\
& \frac{1}{2} K_{S, \text { sup }}\left(e^{-\gamma L_{T}}-e^{\gamma L_{T}}\right)
\end{aligned}
$$

where

$$
\mathrm{K}=\left[\left(\mathrm{CZ} Z_{\mathrm{TS}}-\left[\mathrm{ZZ}_{\mathrm{C}}\right) /\left(\mathrm{AZ} \mathrm{SS}_{\mathrm{SS}}-\mathrm{BZ} \mathrm{C}\right)\right]^{-1}\right.
$$

$A=e^{-\gamma L S}+e^{\gamma L S}$

$B=e^{-\gamma L S}-e^{y L ;}$

$C=e^{-\gamma L_{T}}+e^{\gamma L_{T}}$

$D=e^{\gamma L_{T}}-e^{-\gamma L T}$

Similarly, when the fault is occurred within $\mathrm{L}_{\mathrm{s}}$, we can intentionally substitute the following relationships:

$V_{S}=V_{R}, V_{R}=V_{S}, I_{S}=-I_{R}$ and $I_{R}=-I_{S}$ into the equation (4). Then, fault location $D^{\prime}$ respecting the reference located at the sending end $(D=L)$ can be easily obtained. The final fault location $\mathrm{D}$ with respect to the 
reference located at receiving end can be computed from $D$ $=\mathrm{L}-\mathrm{D}^{\prime}$.

\subsection{Estimation of Source Impedance}

Due to time-varying property of equivalent source impedance, $Z_{S s}$ and $Z_{S R}$ in Fig.1, the estimation of the source impedance is essential. These can be determined respectively by following equations:

$Z_{\text {sS }}=V_{S, \text { sup }} /\left(-I_{S, \text { sup }}\right)$

$Z_{S R}=V_{R, \text { sup }} / I_{R \text {,sup }}$

\subsection{The SDFT Algorithm}

In order to achieve a high degree of accuracy on locating the fault, it is vitally important to be able to accurately estimate the phasors of fundamental frequency from the measured discrete data. A new digital algorithm based on Discrete Fourier Transform, terms as Smart Discrete Fourier Transform (SDFT), is adopted to meet the purpose. The SDFT not only keeps all of the advantages of DFT but also smartly take frequency deviation from nominal frequency and harmonics into consideration. The readers are encouraged to refer to the paper [8] for detailed description.

\section{Performance Evaluation}

\subsection{Simulation example}

A $161 \mathrm{kV}, 100$ kilometers transposed transmission line tapped a source of generator via a 2 kilometers lines is selected to be a simulation sample for verifying the accuracy of proposed algorithm. The tap is located at the 20 kilometers away from receiving end. Distributed parameters model is adopted to model the transmission line. The transmission line parameters used are as the followings:

Zero-sequence parameters:

$$
\begin{aligned}
& \mathrm{R}_{0}=0.4175 \Omega / \mathrm{km} \\
& \mathrm{L}_{11}=1.644 \times 10^{-3} \quad \mathrm{H} / \mathrm{km} \\
& \mathrm{C}_{0}=22 \times 10^{-9} \quad \mathrm{~F} / \mathrm{km}
\end{aligned}
$$

Positive-sequence parameters:

$$
\begin{aligned}
& \mathrm{R}_{1}=0.0385 \Omega / \mathrm{km} \\
& \mathrm{L}_{1}=0.5676 \times 10^{-3} \mathrm{H} / \mathrm{km} \\
& \mathrm{C}_{1}=38 \times 10^{-9} \mathrm{~F} / \mathrm{km}
\end{aligned}
$$

*Conductance is neglected in the simulation.

Both ends of the line are replaced by Thevenin's equivalent, that is, an equivalent source in series with source impedance. The proposed algorithm was evaluated by the data obtained from the ATP simulator.

In our simulation cases, the total time of simulation is $\mathrm{T}=$ 200 (m-sec), and the data is sampled at sampling rate of 3.84 $\mathrm{kHz}$. The error of locating fault is expressed in terms of a percentage of the total line length, i.e.

$$
\% \text { error }=\frac{\text { Estimated Location }- \text { Actual Location }}{\text { Total Line Length }} \times 100 \%(10)
$$

\subsection{Simulation Results}

Table. 1 shows the simulation results of fault location under different fault types and fault locations. The simulation results show that the maximum error of fault location estimation is less than $0.3 \%$ under all conditions. This means that the errors of fault location estimation are well within 300 meters (about a span of two transmission line towers at Taipower system). Table. 2 shows the simulation results under different pre-fault load conditions. The fault type of testing is single-phase ground fault. The pre-fault load conditions are determined by adjusting the difference of the phase angle between the two Thevenin's equivalent sources at both ends of transmission lines. An examination of table. 2 indicates the pre-fault load conditions cause little effect on the accuracy of fault location. Table. 3 show the resuits of fault location under source impedance varying from 0.2 to 5 times of original value. It illustrates the fact that the proposed algorithms are independent of variation of source impedance outside the considered lines.

\subsection{Sensitivity study}

All the simulation results described above are based on a assumption that the source impedance of generator tapped to the existing transmission lines can be accurately obtained. In practice, those parameters may be inaccurate. Therefore, it is necessary to study the sensitivity of the proposed algorithm to the error of source impedance. Table. 4 presents the percentage of error caused by the setting errors of the source impedance of generator tapped to the existing transmission lines. The average error caused by a $20 \%$ variation of source impedance is approximately equal to $1.34 \%$.

\section{CONCLUSION}

New rights of way for transmission lines are difficult to obtain. In some case, a tapping transmission lines are the only reasonable solution to a given problem. So, a transmission lines tapped with a source of generator will happen more frequently. This paper presents a novel faulted section discrimination index and fault location algorithm for locating fault on transmission lines tapped with a source of generator. The simulation results have verified the validity of the proposed algorithm. The maximum error of fault location estimation is less than $0.3 \%$ under different fault types, fault resistance, pre-fault load conditions and different equivalent source impedance. 


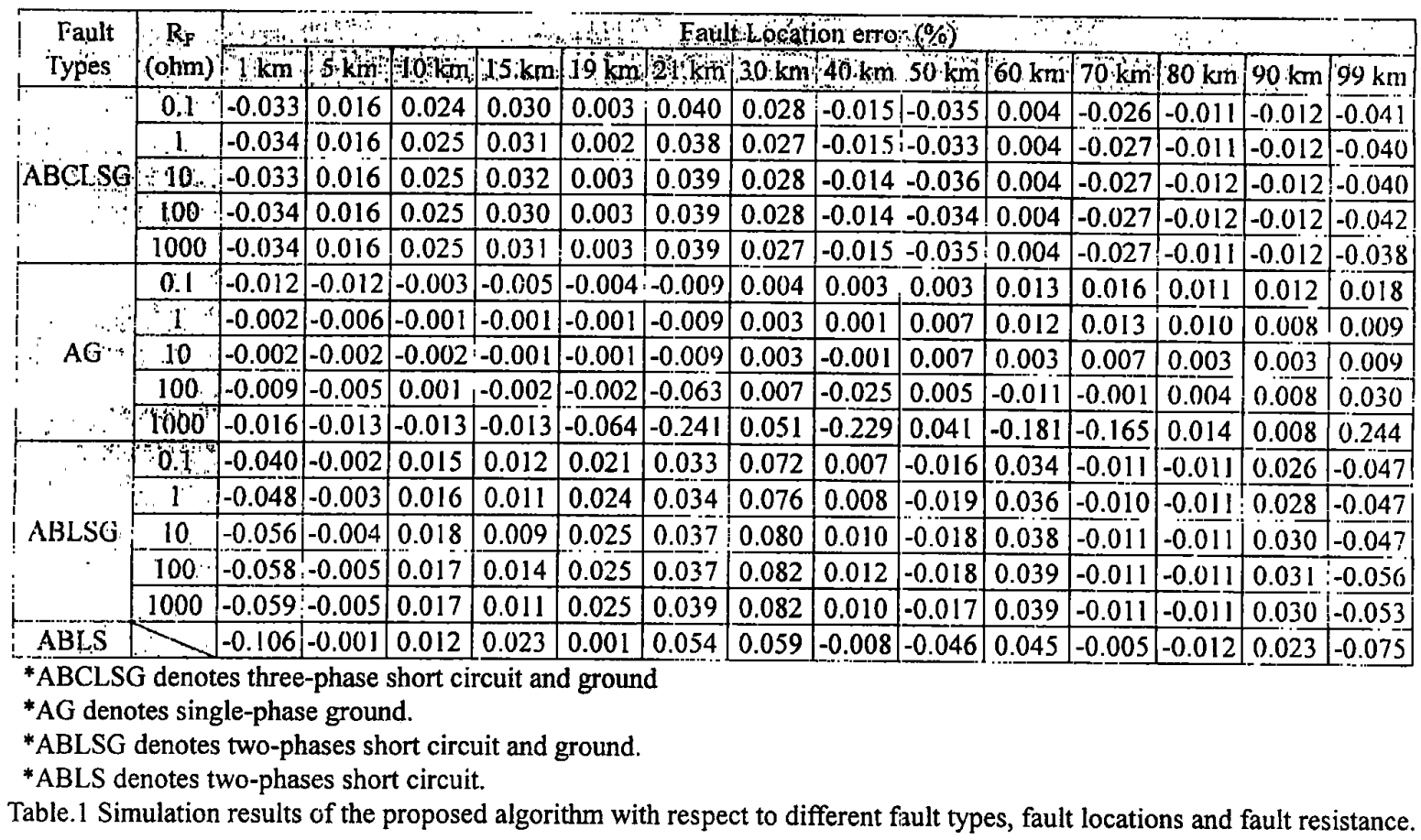

\begin{tabular}{|c|c|c|c|c|}
\hline Phase & \multicolumn{4}{|c|}{ Fault-Eocation error (\%) } \\
\hline $\begin{array}{l}\text { Angle } \\
\text { Difference } \\
\text { (degrces) }\end{array}$ & $5 \mathrm{~km}$ & $15 \mathrm{~km}$ & $45 \mathrm{~km}$ & $90 \mathrm{~km}$ \\
\hline 10 & -0.012 & -0.005 & 0.010 & 0.012 \\
\hline 20 & -0.011 & -0.002 & 0.009 & 0.011 \\
\hline 30 & -0.011 & -0.001 & 0.008 & 0.011 \\
\hline-30 & -0.012 & -0.004 & 0.010 & 0.007 \\
\hline
\end{tabular}

Table. 2 Simulation results under different pre-fault load conditions

\begin{tabular}{|c|c|c|c|c|}
\hline $\begin{array}{c}\text { Variation. } \\
\text { of Source } \\
\text { Impedance }\end{array}$ & \multicolumn{4}{|c|}{ Fatilt Location error (\%) } \\
\cline { 2 - 5 } & $5 \mathrm{~km}$ & $15 \mathrm{~km}$ & $45 \mathrm{~km}$ & $90 \mathrm{~km}$ \\
\hline 0.2 time & -0.001 & -0.001 & 0.022 & 0.027 \\
\hline 0.5 time & -0.002 & -0.002 & 0.020 & 0.039 \\
\hline 2 times & -0.003 & 0.002 & 0.012 & -0.002 \\
\hline 5 times & -0.007 & -0.011 & 0.015 & 0.014 \\
\hline
\end{tabular}

Table. 3 fault location results under source impedance varying from 0.2 to 5 times of original value.

\begin{tabular}{|c|c|c|c|c|}
\hline \multirow{2}{*}{$\begin{array}{l}\text { Error (\%) } \\
\text { of Setting: } \\
\text { Value }\end{array}$} & \multicolumn{4}{|c|}{ Fault Location errof $(\%)$} \\
\hline & $\mathrm{S} \mathrm{km}$ & $1.5 \mathrm{~km}$ & $45 \mathrm{~km}$ & $90 \mathrm{~km}$ \\
\hline$-20 \%$ & -1.131 & -0.509 & 2.136 & 2.438 \\
\hline$-10 \%$ & -0.519 & -0.233 & 0.977 & 1.145 \\
\hline $10 \%$ & 0.421 & 0.183 & -0.812 & -0.986 \\
\hline $20 \%$ & 0.8057 & 0.345 & $-1,514$ & -1.876 \\
\hline
\end{tabular}

Table. 4 The percentage of errors caused by the setting error of the source impedance

\section{REFERENCES}

[1] M. Sachdev and R. Agarwal, "A technique for estimating transmission line fault location from digital impedance relay measurment" IEEE Transaction on Power Delivery, Vol.3, No.1, January 1988, pp.121-129.

[2] D. J. Lawrence, L. Cabeza, and L, Hochberg, "Development of an Advanced Transmission Line Fault Location System Part II- Algorithm Development and Simulation," IEEE Transaction on Power Delivery, Vol.7, No.4, October 1992,pp. 1972-1983.

[3] A. A.Girgis, D. G. Hart, and W. Peterson, "A new Fault Location Technique for Two- and Three Terminal Lines", IEEE Transaction on Power Delivery, Vol. 7, No.1, January 1992, pp.98-107.

[4] T. T. Takagi, et al," Development of a New Fault Locator Using the One-Terminal Voltage and Current Data," IEEE Transaction on PAS, Vol. PAS-101, No. 8, August 1982, pp.2892-2898.

[5] L. Eriksson, M. Saha, and G.D. Rockfeller, "An Accurate Fault Locator with Compensation for Apparent Reactance: in the Fault Resistance Resulting from Remote-End infeed", IEEE Transaction on PAS, Vol. PAS-104, No.2, February 1985, pp.424-436.

[6] J.-A Jiang, Y.-H. Lin, C.-W. Liu, J.-Z. Yang, and T.-M. Too, "An Adaptive Fault Locator System for Transmission Lines", IEEE/PES summer meeting, July 1999, pp.930-936

[7] J.-A Jiang, J.-Z. Yang, Y.-H. Lin, C.-W. Liu, J.-C. Ma, "An Adaptive PMU Based Fault Detection/ Location for Transmission Lines: Part I: Theory and Algorithms", IEEE Transaction on Power Delivery, PE-017PRD (09-99).

[8] J.-Z. Yang and C.-W. Liu, "A precise Calculation of Power Frequency and Phasor", IEEE Transaction on Power Delivery, PE-020PRD (09-99). 


\section{BIOGRAPHIES}

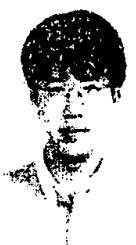

Ying-Hong Lin was born in Taipei, Taiwan, in 1970. He received his B.S. degree in electrical engineering from Taiwan University of Technology in 1995, and M.S. degree in National Taiwan University in 1999. He is presently a graduate student in the electrical engineering department, National Taiwan University, Taipei, Taiwan. His interested researches are the application of GPS and PMU in power system

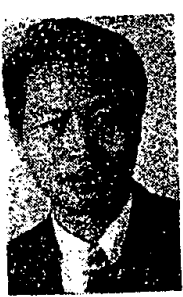

Chih-Wen Liu was born in Taiwan in 1964. He received the B.S. degree in Electrical Engineering from National Taiwan University in 1987, and M.S. and Ph.D. degrees in electrical engineering from Cornell University in 1992 and 1994. Since 1994, he has been with National Taiwan University, where he is associate professor of electrical engineering. He is a member of the IEEE and serves as a reviewer for IEEE Transactions on Circuits and Systems, Part I. His main research area is in application of computer technology to power system monitoring, operation, protection and control. His other research interests include GPS time transfer and chaotic dynamics and their application to system problems.

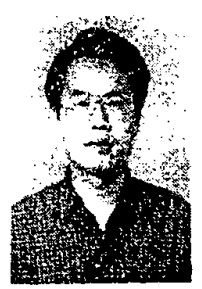

Joe-Air Jiang was born in Tainei, Taiwan, in 1963. He graduated from National Taipei University. of Technology in 1983 and received M.S. and Ph.D degrees in electrical engineering from National Taiwan University, Taipei, Taiwan in 1990 and 1999. Since 1990, he has been with Private Kuan-Wu Institute of Technology and Commerce where he is assist professor of electrical engineering. His area of interest is computer relaying and bio-effects of EM-wave.

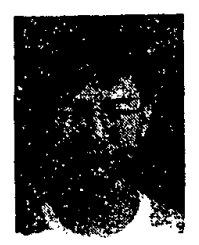

Jun-Zhe Yan was born in Tainan, Taiwan, in 1971. He received his B.S. degree in electrical engineering from Tatung Institute of Technology in 1992 and M.S. degree from National Taiwan University in 1995 . He is presently a graduate student in the electrical engineering department, National Taiwan University, Taipei, Taiwan. 